\title{
Optimized Tracing of iCF-enabled Programmable Data Planes
}

\author{
Lucas Castanheira \\ lbcastanheira@inf.ufrgs.br \\ Institute of Informatics, UFRGS
}

\author{
Alberto Schaeffer-Filho \\ alberto@inf.ufrgs.br \\ Institute of Informatics, UFRGS
}

\author{
Theophilus A. Benson \\ tab@cs.brown.edu \\ Brown University
}

\begin{abstract}
Programmable data planes (PDPs) allow for processing functionality to be placed inside the network, in the form of in-network compute functions (iCFs). However, the crossing over from server into the more constrained PDP devices makes diagnosis challenging.

We present the design of a tracing framework, which extends server tracing into PDPs. This framework uses annotations to collect and encapsulate trace data into a tracing abstraction, and offers a query language with a high-level interface for performing usual tracing tasks. Finally, we employ domain-specific optimizations, co-designing hardware and software, for trace data aggregation and processing.
\end{abstract}

\section{CCS CONCEPTS}

- Networks $\rightarrow$ Programmable networks; In-network processing; Network management; Network monitoring; Programming interfaces.

\section{KEYWORDS}

iCF, Tracing, Debugging, Telemetry, Programmable Networks

\section{INTRODUCTION}

In-network compute has emerged as a means to improve traditional applications by allowing parts of them to run inside network devices. Works exploring the use of iCFs have seen promising improvements in performance and scalability [4, 6]. However, these hybrid servernetwork solutions create management shortcomings [1] such as the breakage of diagnosis tools, e.g., distributed tracing, which are currently unsupported by PDPs. For iCF deployments to thrive, we must bridge the gap between iCF functionality and diagnosis.

Although PDPs already have standalone debugging tools, these are usually device-centric and therefore ill-equipped to provide the holistic, end-to-end diagnosis that is expected of servers. Current debug tools will ultimately not be able to answer the questions that operators ask at runtime to troubleshoot their systems (generally related to causation and performance). For iCFs to attain this high level of observability, we must be able to provide a macro view of the (cross-plane) components interacting (e.g., latency profiling) and also, when needed, a fine-grained report over why things happened (e.g., full-fledged trace with context information). Thus, with

Permission to make digital or hard copies of part or all of this work for personal or classroom use is granted without fee provided that copies are not made or distributed for profit or commercial advantage and that copies bear this notice and the full citation on the first page. Copyrights for third-party components of this work must be honored. For all other uses, contact the owner/author(s).

SIGCOMM '20 Demos and Posters, August 10-14, 2020, Virtual Event, USA

(C) 2020 Copyright held by the owner/author(s)

ACM ISBN 978-1-4503-8048-5/20/08 . \$ \$15.00

https://doi.org/10.1145/3405837.3411376 the intent of aiding the graceful integration of iCFs into server computation, our focus is to implement distributed tracing in PDPs.

To extend tracing into the data plane, we draw inspiration from recent network monitoring solutions $[3,5]$ and rethink tracing as a language-directed process. We implement a query language which can express a comprehensive set of both usual and novel tracing tasks. On top of that language directed paradigm, we present several domain-specific optimizations for reducing operational overheads.

\section{OVERVIEW}

Figure 1 illustrates the workflow of our tracing framework. The first steps are designating what data should be traced and when. We observe that, today, developers annotate code to control the internal elements that tracing has visibility into. As such, PDP developers using our system will annotate what low level variables should be traced directly in the source code (step 1). As for when to trace that data, we create a shim layer at every server that tags packets with unique identifiers (2), based on runtime conditions. These runtime conditions are established by dynamic operator queries (3). A query specifies, at a high-level, the data it wants traced (e.g., latency), which is then mapped to low-level annotated variables (e.g., timestamps). The code necessary to collect these previously annotated variables is then automatically compiled into the switches (4). Typically the shim layer will tag packets that pertain to a server execution that is already being traced. The tagged packet will generate spans at switches where it triggers iCFs (5) with spans being collected and integrated into server traces (6).

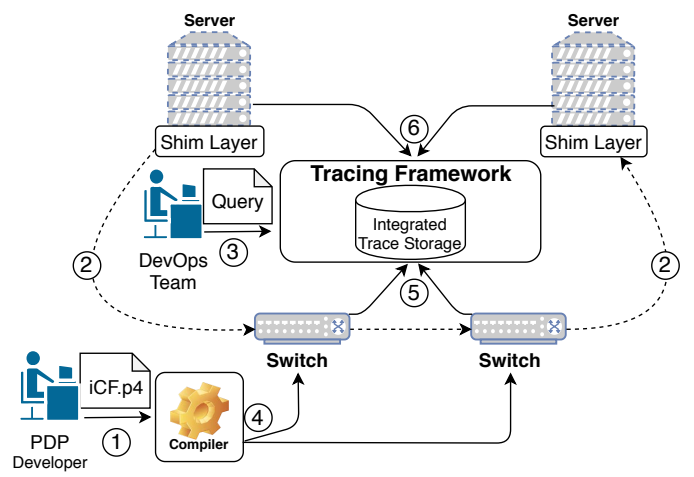

Figure 1: Tracing Workflow

The fundamental challenge in this approach lies in the restrictions placed by the PDP-ecosystem, as PDPs are limited in physical resources. Figure 2 shows common ways to export from the PDP annotated context data for tagged executions. We chose (c) because it does not hog on packet real estate (a) and we can control the intake of traces from software, since letting traffic itself span telemetry traffic (b) can topple the network [2]. 


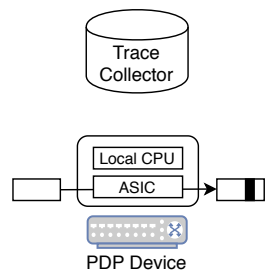

(a)

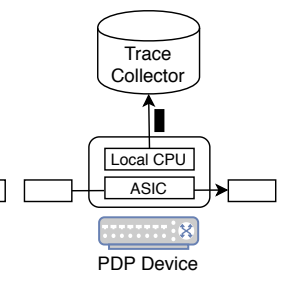

(b)

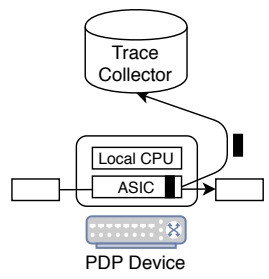

(c)
Figure 2: Design space for Data Plane Tracing: the tracing data is expressed as a black box. This information is either stored in the packet (a); sent in a control packet to the collector (b); or, temporarily stored in memory then forwarded to the collector $(c)$.

While the above strategy is sufficient for including tracing into PDPs, it misses out on several optimizations opportunities and will ultimately create very basic spans with low level PDP variable state. To tackle both of these problems, we incorporate a post-processing into our system. The guiding insight here being that if we know the high level data we wish to present to users we can pre-process this data at the ASIC and the local CPU of switches. For example, we can push simple comparisons to the ASIC itself, which can drop traces that do not match the comparisons at virtually no cost. More complex processing can be done at the local CPU, that can batch spans of a given query, calculate useful metrics that the ASIC cannot, and even perform compression over outbound batches, further reducing communication overheads. This is the first part of our co-design: pushing back processing as far as possible to prune the data on flight and ease processing and storage overheads.

A further optimization is to provide users with annotations that perform aggregation on the ASIC level (data that can be stored on data structures such as counting arrays, matrices, sketches). This stems from the fact that most distributed tracing solutions today include metrics along traces, and previous works have shown that metrics that are easily aggregated in the data plane can be valuable for providing a low-cost macro view of the system. Because sketching is generally lossy for isolated variables, we only allow it for aggregate metrics over multiple packets (e.g., Marple's Linearin-State [5]).

\section{ARCHITECTURE}

We briefly describe the architecture of our system and its main components (Figure 3).

Query Language We design a query language that provides a high-level interface for capturing tracing tasks traditionally applied to distributed tracing. It takes inspiration from the well-known SQL, and incorporates constructs useful for Developer-Operators (DevOps) to specify tracing.

Shim Layer: Our shim layer is placed at every tracing-enabled server. It intercepts iCF invocations, along with context data local to each server, and discovers if the invocation should be tagged for tracing based on the query and other parameters (such as sampling).

Query Compiler: Draws information from the annotations and the query to generate the Storage Layer and configure the PDP agent and shim layer. The query compiler optimizes queries by judiciously decomposing them into local processing on the ASIC (ASIC Pruning), on the PDP agent (Local Pruning) and the part that needs to inevitably run globally on the controller (Global Processing), thereby reducing storage, processing, and bandwidth requirements.

Storage Layer: Our storage layer consists of data structures for capturing and maintaining query specific annotations while addressing PDP hardware-specific limitations. Sometimes data aggregation can be performed efficiently on the data plane, for those cases we expose a series of sketch data structures.

Trace Orchestrator: leverages unique identifiers, fuses PDP traces with their server counterparts, exporting an end-to-end trace.

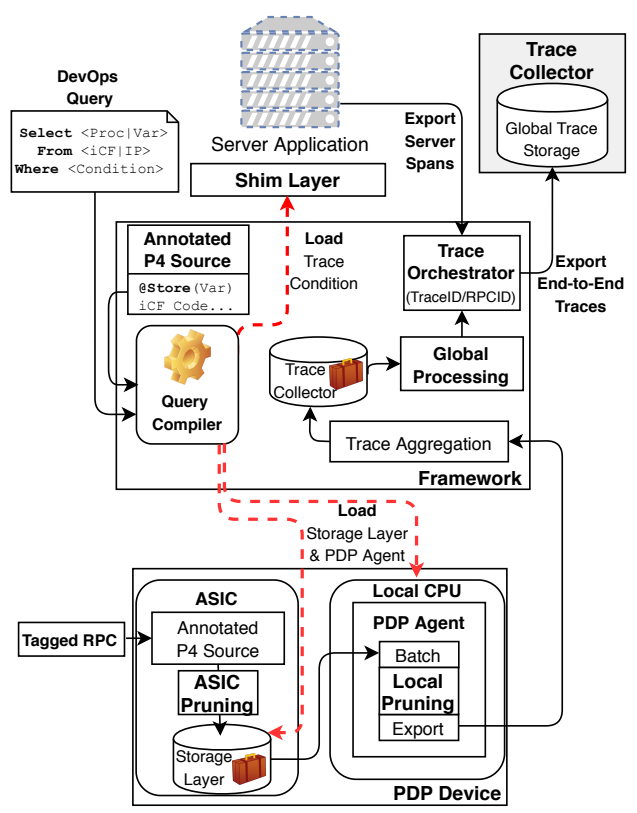

Figure 3: Architecture Diagram

\section{EVALUATION}

Our prototype was implemented for the NetFPGA SUME. Preliminary experiments have shown that the instrumentation of our framework is lightweight in terms of hardware (approximately $1 \%$ of total LUTs and FFs).

\section{CONCLUSION}

We presented the first step in holistic, end-to-end tracing of iCFenabled PDPs. We introduce a language-directed tracing paradigm and leverage $\mathrm{P} 4$ to extend tracing into PDPs and a hardware-software co-design for optimizing it.

\section{ACKNOWLEDGMENTS}

We thank the anonymous reviewers for their very insightful feedback. Theophilus A. Benson would like to thank the NSF for award CNS-1749785. Alberto Schaeffer-Filho would like to thank CNPq for research grant 312091/2018-4. This study was financed in part by the Coordenação de Aperfeiçoamento de Pessoal de Nível Superior - Brasil (CAPES) - Finance Code 001, and also by NSF CNS-1740911 and RNP/CTIC (P4Sec) grants. 


\section{REFERENCES}

[1] T. A. Benson. In-network compute: Considered armed and dangerous. HotOS '19.

[2] Cisco. Cisco IOS NetFlow - a technical overview. https://www.cisco.com/ c/en/us/products/collateral/ios-nx-os-software/ios-netflow/prod_white_ paper0900aecd80406232.html. Last accessed in Feb 17, 2019.

[3] A. Gupta, R. Harrison, M. Canini, N. Feamster, J. Rexford, and W. Willinger. Sonata: Query-driven streaming network telemetry. SIGCOMM '18.
[4] X. Jin, X. Li, H. Zhang, R. Soulé, J. Lee, N. Foster, C. Kim, and I. Stoica. Netcache: Balancing key-value stores with fast in-network caching. SOSP '17.

[5] S. Narayana, A. Sivaraman, V. Nathan, P. Goyal, V. Arun, M. Alizadeh, V. Jeyakumar, and C. Kim. Language-directed hardware design for network performance monitoring. SIGCOMM '17, 2017.

[6] A. Sapio, I. Abdelaziz, A. Aldilaijan, M. Canini, and P. Kalnis. In-network computation is a dumb idea whose time has come. HotNets-XVI, 2017. 